\title{
PRODUCTION OF HEAT RESISTANT COMPOSITE BASED ON SILOXANE ELASTOMER AND MULTIWALL CARBON NANOTUBES
}

\author{
I.V. Bessonov ${ }^{1}$, N.V. Karelina ${ }^{1}$, M.N. Kopitsyna ${ }^{1}$, A.S. Morozov ${ }^{1}$, S.V. Reznik ${ }^{1 a}$, V. Yu. Skidchenko ${ }^{1}$ \\ ${ }^{1}$ Bauman Moscow State Technical University, 105005 Moscow, Russia
}

\begin{abstract}
Development of a new generation of composite with unique thermal properties is an important task in the fields of science and technology where material is operated at high temperatures and exposure to a short-wave radiation. Recent studies show that carbon nanomaterials (fullerenes and carbon nanotubes) could improve the thermal, radiation and thermal-oxidative stability of the polymer matrix. In this article the development of a new heat resistant composite based on elastomer and carbon nanotubes (CNT) was performed and physicochemical properties of final product were evaluated.
\end{abstract}

\section{Introduction}

Various high-tech areas like modern aerospace engineering impose several restrictions on thermal stability of elastomeric materials. Among different heat resistant elastomeric matrices polysiloxanes are the best candidates due to its flexible and thermally stable atom chain Si-O-Si [1,2].

This work is aimed in production of heat resistant composite based on siloxane elastomer and multiwall carbon nanotubes (MCNT). Carbon nanotubes are applied as they possess the necessary property of increasing composite's resistance to high temperatures and ionizing radiation [3].

\section{Composite production and investigation}

Due to the fact that selected siloxane elastomer is a viscous liquid, it was necessary to previously disperse nanotubes in a solvent followed by impregnation of dispersion in the polymer matrix.

Organic solvents are widely used for dispersing nanotubes in a liquid medium [4]. We decided to produce a series of experimental samples to choose the optimal solvent. Following solvents were selected for composite preparation: ethanol, chloroform, dichloromethane, toluene and 1,2dichloroethane. All of them could dissolve siloxane elastomer and could be easily evaporated after mixing with the dispersion of MCNTs.

Mixture of MCNTs in solvent $(10 \mathrm{ml})$ was dispersed applying two different procedures, such as ultrasonication and mechanical stirring. Next the dispersion was introduced into a siloxane matrix. Ultrasonication was found to be more effective, since only this method allowed achieving a stable

${ }^{a}$ Corresponding author: sreznik@bmstu.ru 
emulsion without sedimentation within 24 hours. Minimum dispersion time with no visible sedimentation during this period in 1,2-dichloroethane was 20 minutes.

The resulting composites were characterized by infrared (IR) spectroscopy, thermogravimetric analysis (TGA) and scanning electron microscopy (SEM). IR spectra are shown in Figure 1. There is no significant difference in the spectrum of pure siloxane and composites on the basis of siloxane and MCNT, which proves that no solvent left in the composite.

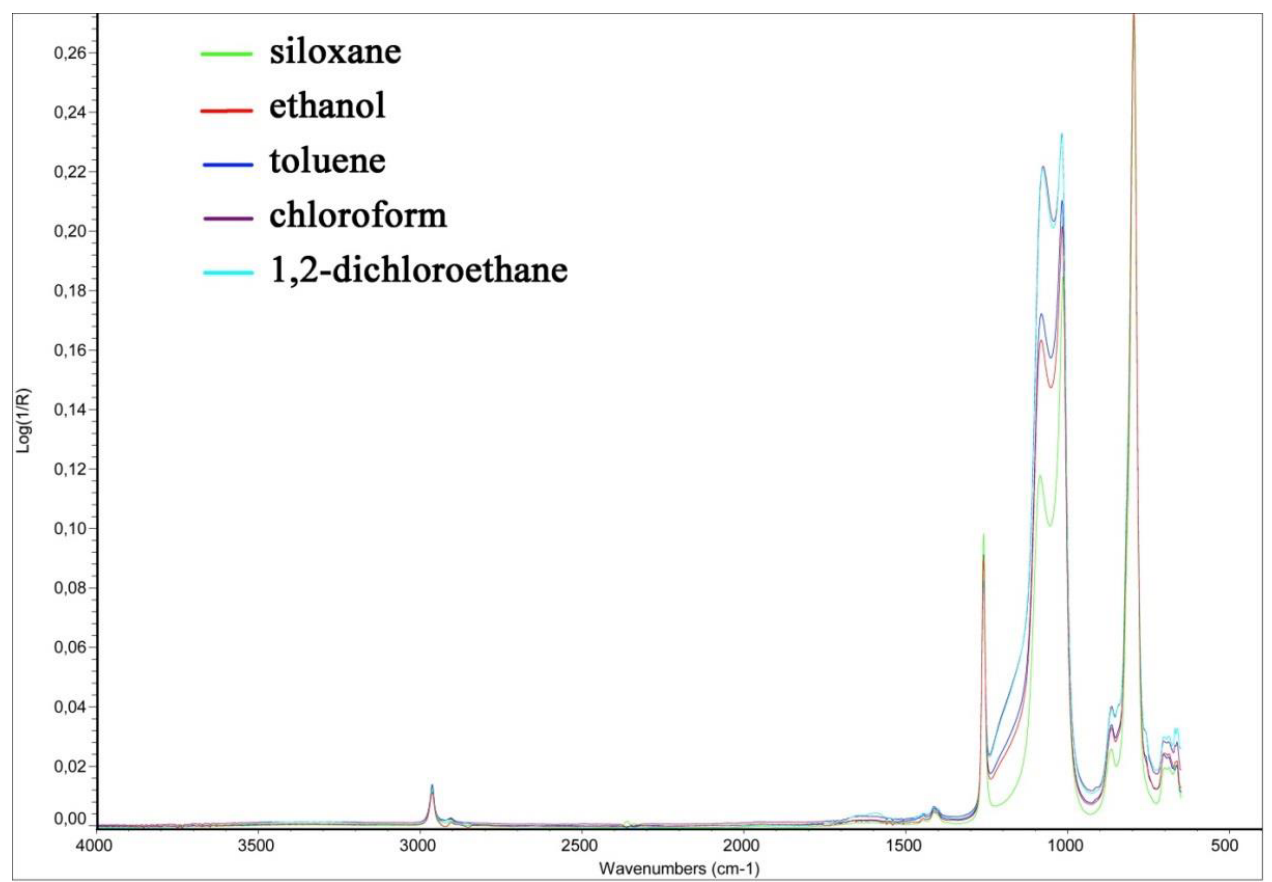

Figure 1. IR spectra of the siloxane and composites on the basis thereof.

Thermogravimetric measurements were carried out in the temperature range from 25 to $400{ }^{\circ} \mathrm{C}$, heating rate $10 \mathrm{~K} / \mathrm{min}$ in an inert atmosphere (argon). All achieved composites possessed rather high thermal stability. Among others solvents 1,2-dichloroethane turned out to be the most effective dispersing agent with the lowest mass loss $2,2 \%$ in the studied temperature range (Fig. 2). 


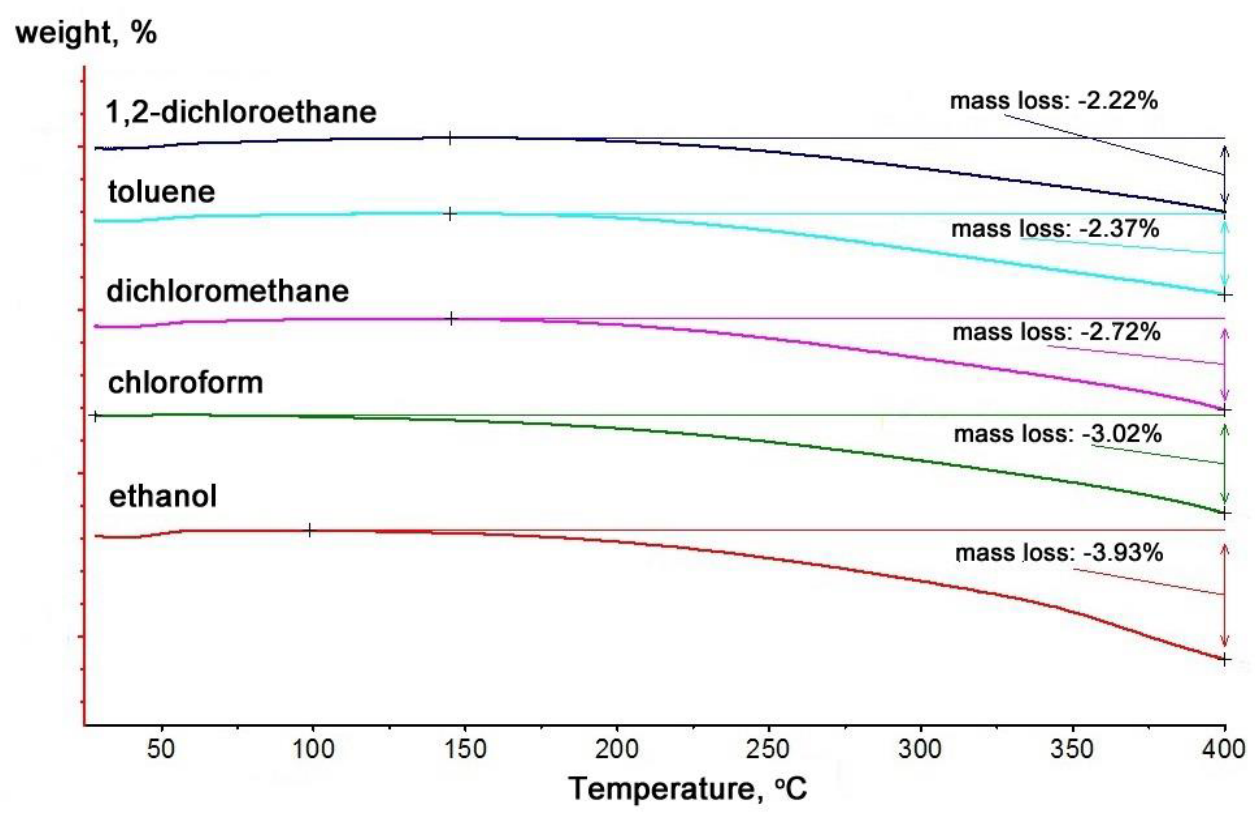

Figure 2. TGA spectra of composites on the basis of siloxane oligomer.

Heat resistance of composite obtained by dispersing MCNTs in 1,2-dichloroethane and siloxane oligomer without filler was compared applying thermogravimetric analysis (Fig.3). It was shown that addition of MCNT's dispersion in selected solvent significantly increases the thermal stability of composite. Pure siloxane losses $3 \%$ of its mass at $390^{\circ} \mathrm{C}$, while for the composite this temperature is higher and equals $436{ }^{\circ} \mathrm{C}$. Thus thermal degradation temperature for composite exceeds temperature of pristine elastomer by $46^{\circ} \mathrm{C}$.

Table 1.

\begin{tabular}{|c|c|c|}
\hline Sample & $\begin{array}{c}\Delta \mathrm{m}=1 \% \\
\text { Temperature, }{ }^{\circ} \mathrm{C}\end{array}$ & $\begin{array}{c}\Delta \mathrm{m}=3 \% \\
\text { Temperature, }{ }^{\circ} \mathrm{C}\end{array}$ \\
\hline Pure siloxane & 301 & 390 \\
\hline Composite with MCNT & 336 & 436 \\
\hline
\end{tabular}




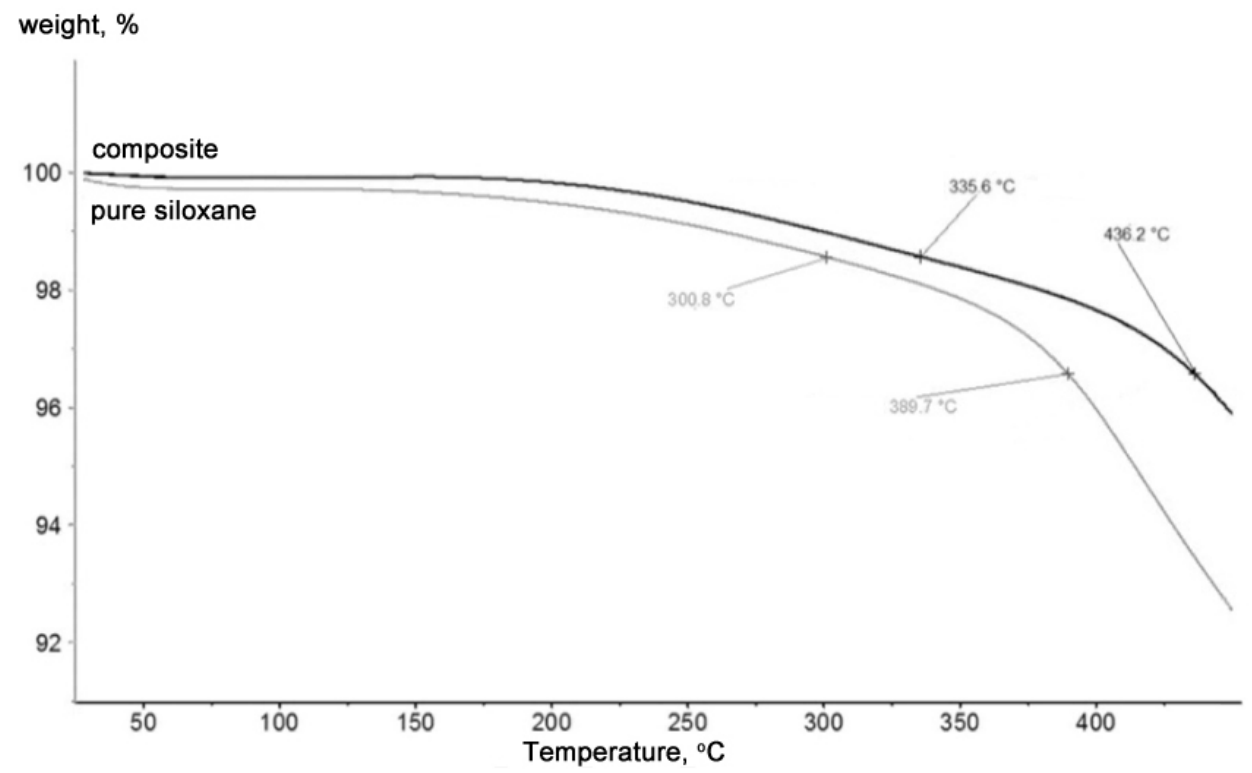

Figure 3. TGA spectra of the siloxane (red) and composite (blue) on the basis thereof.

Uniform distribution of MCNT in the siloxane matrix was confirmed by SEM. The images are shown in Figure 4.
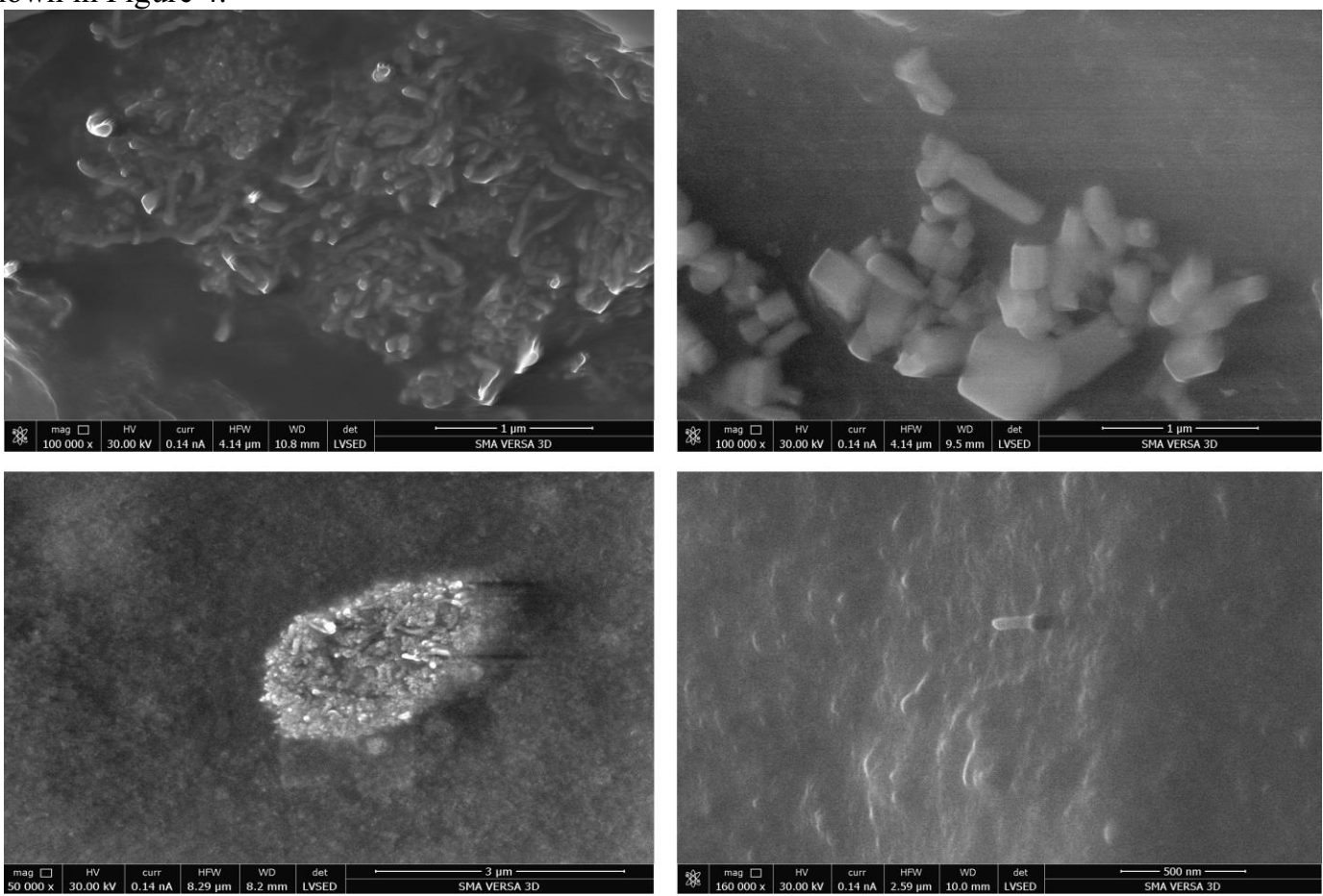

Figure 4. SEM images of composites, MCNT dispersion was carried out in ethanol (top left), toluene (top right), chloroform (bottom left) and 1,2-dichloroethane (bottom right).

It was determined that ethanol and toluene are not suitable for dispersion, as most MCNT aggregate on a surface as large agglomerates of various size and form. Chloroform application allows 
achieving more or less uniform distribution of nanotubes, but still there are individual agglomerates of considerable sizes (figure 4, down left). Finally, applying 1,2-dichloroethane leads to effective dispersion and composite manufacture with uniform distribution of MCNTs without the formation of visible agglomerates.

\section{Conclusion}

A novel heat resistant composite based on siloxane matrix and carbon nanotubes was produced and its physicochemical properties were assessed. High quality dispersion of nanotubes was achieved through extensive testing of a variety of widely used solvents and variation of dispersing procedures. Thermal stability of composite was shown to be $46{ }^{\circ} \mathrm{C}$ higher than that of pristine elastomer.

The work leading to these results has received funding from the Ministry of Education and Science of the Russian Federation (№ 14.577.21.0095 from 25th August 2014). Unique dentifier RFMEFI57714X0095.

\section{References}

1. Z. Li, S. Nambiar, W. Zheng, J.T.W. Yeow, Materials Letters, 108, 79 (2013)

2. X. Shi, B. Jiang, J. Wang, Y. Yang, Carbon, 50, 1005 (2012)

3. H. Lorenz, J. Fritzsche, A. Das, K.W. Stockelhuber, R. Jurk, G. Heinrich, M. Kluppel, Composites Science and Technology, 69, 2135 (2009)

4. D.S. Kim, D. Nepal, K.E. Small, Individualization of Single-Walled Carbon Nanotubes: Is the Solvent Important? 1, 1117 (2005) 